\title{
Auxiliary field simulation and Coulomb's Law
}

\author{
A.C. Maggs ${ }^{\text {a }}$, J. Rottler b \\ ${ }^{a}$ Laboratoire de Physico-Chimie Théorique, UMR CNRS-ESPCI 7083, 10 rue Vauquelin, F-75231 Paris Cedex 05, France. \\ b Princeton Institute for the Science and Technology of Materials (PRISM), Princeton University, Princeton, NJ 08544, \\ $U S A$.
}

\begin{abstract}
We review a family of local algorithms that permit the simulation of charged particles with purely local dynamics. Molecular dynamics formulations lead to discretizations similar to those of "particle in cell" methods in plasma physics. We show how to formulate a local Monte-Carlo algorithm in the presence of the long ranged Coulomb interaction. We compare the efficiencies of our molecular dynamics and Monte-Carlo codes.
\end{abstract}

Key words: Monte-Carlo, particle in cell, molecular dynamics, Maxwell's equations, constrained dynamic systems, Coulomb PACS: 02.70.Ns 41.20.Cv 02.70.Uu 77.22.-d 02.50.Ng 02.60.Ed

\section{Introduction}

Computer modeling of charged systems is demanding due to the range of the Coulomb interaction [1]. The direct evaluation of the Coulomb sum for $N$ particles, $U_{c}=\sum_{i<j} e_{i} e_{j} / 4 \pi \epsilon_{0} r_{i j}$, requires computation of the separations $r_{i j}$ between all pairs of particles, which implies $O\left(N^{2}\right)$ operations are needed per sweep or time step. Most large scale codes in use at the moment find the electrostatic energy and forces using the fast Fourier transform [2] after interpolating charges to a mesh. For an ensemble of charges interpolated to $O(M)$ nodes of a lattice this gives the electrostatic energy in a time which scales as $O(N+M \ln M)$ on a mono-processor machine.

It is rather surprising that methods that are based on solution of the Poisson equation are so dominant in mesh based molecular dynamics. In plasma physics, one may use a global solver or al- ternatively one integrates the full local Maxwell equations for the electromagnetic field. We shall show in this article that a similar approach based on Maxwell's equations also gives good results in molecular dynamics; Coulomb's law is implied by Maxwell's equations in the quasi-static limit. The advantage of this approach is that parallel implementations which exhibit $O(N+M)$ scaling are rather easy to write.

After abstracting the part of Maxwell's equations which is essential for Coulomb's law we introduce a local Monte-Carlo algorithm based on an auxiliary electric field $\mathbf{E}$. This method also requires an effort of $O(N+M)$ per sweep and leads to substantial speedups compared to conventional molecular dynamics methods. Again the algorithm is easily parallelizable. Introduction of cluster moves for the field $\mathbf{E}$ give further increases in efficiency in the $O(M)$ mesh work; this is most useful when $N / M$ is small. 


\section{Particle in Cell codes in Plasma Physics}

Particle in cell methods [3,4] are widely used in the simulation of plasmas coupled to Maxwell's equations. Applications are widespread and include astrophysics, laser physics and fusion research. In these algorithms the continuum magnetic and electric fields are discretized on mesh. Particles, however, still move in the continuum:just like mesh based molecular dynamics algorithms. The principle technical difficulty lies in correctly coupling the field and particle degrees of freedom. The coupling is usually performed by interpolation of both the electric charges and the electric currents to the mesh and then by a back interpolating the electrodynamic forces from the mesh onto the particles.

Typically the charge of the particles, $e_{i}$ is interpolated to the nodes while the current, $\mathbf{J}_{i}=\mathbf{v}_{i} e_{i}$ is assigned to the links. This double interpolation is difficult; conservations laws may not be preserved. The importance of exact implementation of conservation laws is now widely recognized from the field of geometric integration [5]. Many charge/current interpolation schemes do not satisfy the continuity equation

$$
\frac{\partial \rho}{\partial t}+\operatorname{div} \mathbf{J}=0
$$

We shall show below that the breakdown of continuity leads to errors in the electrostatic interactions between charges.

Until now the principle solutions to this problem of charge continuity were either the use of a low order (and thus noisy) interpolation scheme for which continuity is exact [3] or the use of higher order schemes with frequent global corrections in which the longitudinal field components are regularly thrown out and then reinitialized from solution of the Poisson equation. A recent and interesting hybrid approach considers modifications to Maxwell's equations [6,7] which stabilize their solutions even in the presence of inconsistencies in the interpolation scheme.

The use of a lattice to interpolate the field degrees of freedom leads to two other sources of error in the energy of a system of charged particles in the quasi-static limit. Firstly the pair interaction be- tween two particles is given by a lattice Green function, rather than the exact interaction in $1 / r$. In the simplest discretizations this leads to an interaction potential of the form $1 / r+O\left(a^{2} / r^{3}\right)$ where $a$ is the lattice spacing. A second and more serious artefact is the variation of the self-energy of the particles as a function of their position with respect to the lattice. This leads to a periodic 1body potential for a particle $i$ with an amplitude which varies as $e_{i}^{2} / \epsilon_{0} a$ [8]: As the lattice spacing decreases this energy variation diverges so the discretization becomes worse. In the plasma literature this 1-body energy is often ignored, one simply uses a very coarse mesh with large $a$ so that the kinetic energy is large compared with this energy scale; in a thermalized plasma the mesh size must be larger than the Bjerrum length, $l_{b}=e^{2} / 4 \pi \epsilon k_{B} T$.

In mesh based molecular dynamics codes these two artefacts in the energy are also present. Usually the errors are reduced by convolving the charge of each particle over a large number of lattice points $[2,9,10]$, or equivalently by multiplying the structure factor in Fourier space by an appropriate shaping factor. We need to implement similar methods if we wish to use particle in cell techniques for molecular dynamics.

\subsection{Implementation for Molecular Dynamics}

Recently two groups have implemented a molecular dynamics algorithm based on particle in cell discretizations. As noted above conventional formulations are not quite good enough for direct application in molecular dynamics. This new work has

- Shown how Maxwell's equations give Coulomb's law

- Led to new charge conserving integrators

- Controled lattice artefacts in the energy

In our own work [11] we used interpolation algorithms based on polynomial splines in order to 'tune' the error in the 1-body and pair potentials in a manner very similar to that used in conventional molecular dynamics codes based on Poisson solvers [9]. To do this we generalized the charge conserving algorithm of [3], valid for linear interpolation to general B-spline interpolation. The re- 
sulting algorithm [11] conserves charge to machine accuracy for finite time steps and is time reversible. In order to simplify the implementation we chose to drop the Lorentz force from the particle equations of motion which leads to non-Hamilton (and thus non symplectic) dynamics; however the dynamics do still conserve the total volume in the appropriately defined configuration space. This conservation law implies the existence of a Gibbs distribution for the combined field-particle system.

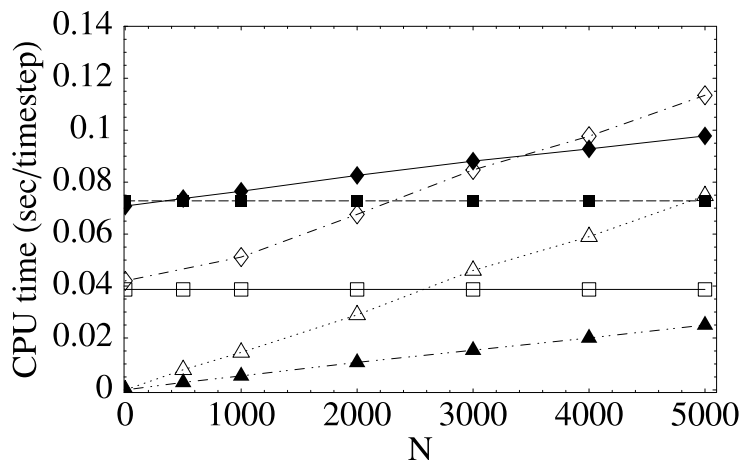

Fig. 1. Cpu time for the field integration $(\square)$, field-particle couplings $(\triangle)$ and total time $(\diamond)$ in a system of size $L=30 a$ as a function of $N$ on an single AMD Athlon CPU. Also shown are results for the same system treated with the P3M method using a charge interpolation of the same order (filled symbols), measures the total time in Fourier based work.

Our molecular dynamics code has been run in two distinct modes. Firstly a mode in which the transverse components of the electric field and the particles are coupled to a thermostat. For this mode we are able to demonstrate, analytically, convergence to a Boltzmann-Gibbs distribution of particles interacting as if with a instantaneous, non-retarded Coulombic interaction. In the second mode we continue to thermostat the particles but anneal the transverse components of the electric field to zero temperature. This mode is very similar to Car-Parrinello [12] simulations in quantum molecular dynamics. Here it is the ratio of the typical particle velocity, $\bar{v}$ to the speed of light, $c$ which is tuned as the optimization parameter. We expect (but here have no proof) that the correct Gibbs distribution is generated when $\bar{v} / c$ is sufficiently small. We again verified numerically that the correct effective interaction is found. A com- parison of the speed of our code compared with a conventional Fourier based Poisson solver [13] is given in Fig. (1). At very low densities our code has a slight advantage, at higher densities the conventional algorithm behaves better. The advantages in each regime however are rather modest.

Pasichnyk and Duenweg [14] have taken a rather different and more direct route to eliminating the errors in the potentials. They make the remark that for low order interpolation schemes one can rather easily calculate the exact numerical value of the 1-body potential. They then perform a direct, numerical subtraction of this energy in their code. They have performed a detailed comparison of this simple and elegant solution with simulations performed with conventional Fourier methods. They find that their code is competitive in both speed and accuracy with conventional methods. The authors work again in the limit $\bar{v} / c$ small but with no thermostating of the electric field. In contrast with our own work they have a full parallel implementation using MPI.

\section{Origin of Coulomb's law}

In electromagnetism Coulomb's law comes [15] from a local expression for the energy: $U=$ $\int \epsilon_{0} \mathbf{E}^{2} / 2 \mathrm{~d}^{3} \mathbf{r}$, and the imposition of Gauss' law $\operatorname{div} \mathbf{E}-\rho / \epsilon_{0}=0$. This motivates the use of the following partition function for the electric field

$\mathcal{Z}(\rho)=\int D \mathbf{E} \prod_{\mathbf{r}} \delta\left(\operatorname{div} \mathbf{E}-\rho / \epsilon_{0}\right) e^{-U / k_{B} T}$.

We change integration variables to $\mathbf{e}=\mathbf{E}+\nabla \phi$ with $\nabla^{2} \phi=-\rho / \epsilon_{0}$ and find

$Z=\int D \mathbf{e} \delta(\operatorname{div} \mathbf{e}) e^{-\beta \frac{\epsilon_{0}}{2} \int(\mathbf{e}-\nabla \phi)^{2} \mathrm{~d}^{3} \mathbf{r}}$.

The cross term in the exponential is zero by integration by parts: $\int \nabla \phi \cdot \mathbf{e} \mathrm{d}^{3} \mathbf{r}=0$, so that

$Z=e^{-\beta \frac{\epsilon_{0}}{2} \int(\nabla \phi)^{2}} \int D \mathbf{e} \delta(\operatorname{div} \mathbf{e}) e^{-\beta \frac{\epsilon_{0}}{2} \int \mathbf{e}^{2} \mathrm{~d}^{3} \mathbf{r}}$

We conclude that $Z=Z_{\text {Coul }} \times$ const, with $Z_{\text {Coul }}$ the partition function of particles interacting with the conventional Coulomb expression: Relative 
statistical weights are unchanged if we allow the transverse field to vary freely, rather than quenching it to zero. To sample the partition function Eq. (2) we have to find solutions to Gauss' law. Integrating Maxwell's equations is one method of continuously and locally updating the system in such a way that Gauss' law is always satisfied.

We now understand the importance of charge conservation in the generation of Coulomb's law: Consider the Maxwell equation

$\epsilon_{0} \frac{\partial \mathbf{E}}{\partial t}=-\mathbf{J}+\operatorname{curl} \mathbf{H}$

and take its divergence, $\operatorname{div}\left(\epsilon_{0} \dot{\mathbf{E}}+\mathbf{J}\right)=0$. If we impose continuity Eq. (1) then we conclude that

$\frac{d}{d t}\left(\epsilon_{0} \operatorname{div} \mathbf{E}-\rho\right)=0$

and Gauss' law is valid. In the absence of continuity Gauss' law is violated and from the above argument Coulomb's law is not generated as the effective thermodynamic interaction between charges.

\section{Monte-Carlo sampling}

As well as performing molecular dynamic sampling of Eq. (2) we have performed Monte-Carlo sampling of the constrained partition function for the case a simple lattice gas [16] and also [8] for offlattice models. These codes work with a mixture of two different Monte-Carlo moves. The first is the motion of a particle together with a slaved update of the electric field in the vicinity of the particle. The second is a grouped update of the electric field on the four links defining a plaquette of the mesh. The two moves are randomly mixed so that the probability of plaquette updates is $p$. These two moves have been related to the two terms on the right hand side of Eq. (5) [17].

We now compare molecular dynamics and Monte-Carlo codes for efficiency: a priori MonteCarlo should be faster.

- One does not restrict step sizes in order to ensure accuracy and stability of the dynamics.

- Energy is easier to calculate than force, particularly when interpolating to and from a mesh.

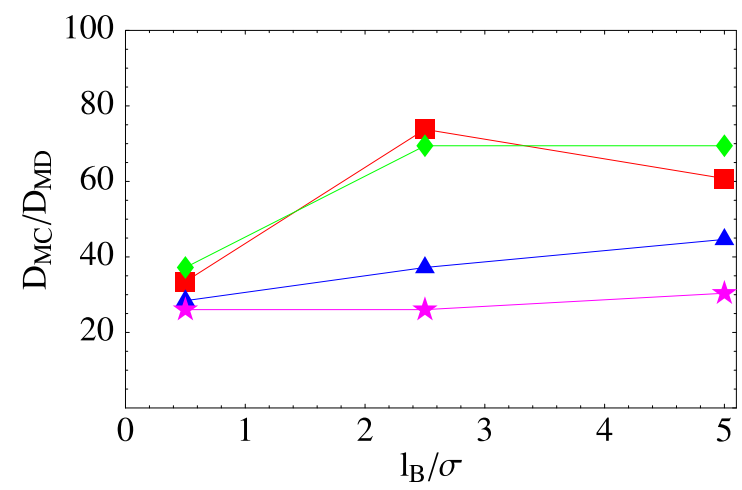

Fig. 2. Ratio of single particle diffusion coefficients in a simple electrolyte obtained from molecular dynamics $D_{M D}$, and Monte Carlo $D_{M C}$; time is measured in sweeps for each code. The particles also interact with a truncated, repulsive Lennard Jones potential of range $r_{c}=2^{1 / 6} \sigma$, where $\sigma=2 a$. Measurements as a function of relative interaction strength for several volume fractions: $\Phi=0.15$ (ם), $\Phi=0.3(\boldsymbol{\diamond}), \Phi=0.45(\boldsymbol{\Delta}), \Phi=0.6(\star)$.

We explore the first point in Fig. (2) by comparing the single particle diffusion coefficients in a simple electrolyte as a function of interaction strength (Bjerrum length) using molecular dynamics parameters from [10]. We also added a truncated, repulsive Lennard Jones potential to prevent overlap of the particles. Simulation time is measured in sweeps, and the interaction strength is varied by changing the Bjerrum length $l_{b}$ from $\sigma / 2$ (weak Coulomb interaction) to $5 \sigma$ (strong interaction). We find that in both codes the diffusivity decreases with increasing $l_{b}$, but the ratio remains roughly constant. At low volume fractions the Monte Carlo code is up to 80 times faster than the molecular dynamics code. This advantage begins to weaken at higher volume fractions, when collisions (due to the LJ potential) between particles slow down the diffusion apparently more strongly in MC. Nevertheless Monte Carlo always retains an advantage. We have not evaluated the second factor in the relative efficiencies since our two codes are structured very differently.

Until now we have only considered media with uniform dielectric properties however Maxwell's equations are valid for arbitrary spatial variation of $\epsilon(\mathbf{r})$. They remain local and thus give an $O(N)$ algorithm for treating implicit solvents in a situation where conventional Fourier codes break down. 
The Monte-Carlo algorithm can also be applied to such media [18]. To do this one works with the electric displacement $\mathbf{D}$ rather than the electric field. The Gauss law constraint now reads $\operatorname{div} \mathbf{D}=\rho$, the local expression for the energy is given by $U=$ $\int \mathbf{D}^{2} / 2 \epsilon(\mathbf{r}) \mathrm{d}^{3} \mathbf{r}$.

\subsection{Effective Monte-Carlo Field Dynamics}

Our Monte-Carlo algorithm is based on local updates to both the particle positions and the electric field. Both the particles and the electric field exhibit diffusive dynamics, in contrast to the propagative behaviour of free particles and waves in the Maxwell equations. We have shown that the field dynamics are given by the Langevin equation [17]:

$\frac{\partial \mathbf{E}}{\partial t}=D\left(\nabla^{2} \mathbf{E}-\operatorname{grad} \rho / \epsilon_{0}\right)-\mathbf{J} / \epsilon_{0}+\vec{\xi}(t)$

which replace Maxwell's equations in our MonteCarlo scheme. Eq. (7) is particularly interesting when there are free, mobile charges so that the electric current is linked to the electric field via the equation $\mathbf{J}=\sigma \mathbf{E}$. In this case the dispersion law of the transverse electric field develops a gap so that $i \omega=\sigma / \epsilon_{0}+D q^{2}$

The relative diffusion rates of the electric and charge degrees of freedom can be adjusted by modifying $p$ and thus $D$. We initially believed that the algorithm should be run in a regime in which the effective diffusion coefficient of the electric field, $D$ is comparable to or somewhat larger to that of the particles: The particles are then always interacting via a field that is close to equilibrium and are not slowed down by the "drag" from the electric degrees of freedom.

Numerical experimentation has shown that one should use a much lower rate of update. There are several ways of understanding this surprising efficiency of the code

- The gap in the electric dispersion law gives a fast relaxation of the electric degrees of freedom even at very long wavelengths.

- In certain limits Coulomb interactions are generated even if the plaquette updates are suppressed entirely $(p=0)$ : a most surprising and deep result linked with the statistical mechanics of discrete plaquette models [19].

\subsection{Cluster Monte-Carlo for $\mathbf{E}$}

The efficiency of the Monte-Carlo algorithm is in part due to the gap which occurs in the dispersion law for the transverse electric field. This gap has as its origin the finite conductivity of a system with a finite density of free charges. What happens when this density is small or zero? Are we obliged to spend much more of our computer budget updating the plaquettes in order to increase $D$ and thus sample the $O(M)$ electric degrees of freedom? For $N / M$ small this would reduce the effective efficiency of the algorithm. Recently we have found a way [20] of reintroducing a gap in the electric field spectrum even when $\sigma=0$ based on ideas used to accelerate the simulation of quantum spin models [19]. This quantum algorithm is in turn inspired by the Swendsen-Wang algorithm for the Ising model. In the language of electrostatics this new cluster algorithm works by nucleating a pair of virtual particles of opposite sign. These particles then evolve by kinetic Monte-Carlo until they annihilate taking the system back to a state of no free particles. The virtual particles re-establish the gap in the electric spectrum and lead to resampling of the electric field in $O(1)$ sweeps. The algorithm gives a dynamic exponent for the field $z=0$, rather than $z=2$ which is implied by Eq. (7) when $\sigma=0$.

\section{Perspectives}

We have introduced a series of algorithms for the local simulation of particles interacting via electrostatic interactions. Molecular dynamics implementations are broadly similar in performance to conventional Fourier based solvers. They can, however, be trivially parallelized in an environment with limited interprocessor bandwidth; this is much harder to do with Fourier based solvers.

We have also formulated an off-lattice MonteCarlo algorithm that is faster than the molecular dynamics equivalent while keeping the advantage of locality. Finally analogies with "worm" or quantum cluster algorithms allow one to integrate over the field degrees of freedom with remarkable efficiency. 
We note that the above discretizations of electrostatics are very similar to those introduced in the 19'th century. At this time there was a very poor intuition as to the physical content and implications of Maxwell's equations. FitzGerald introduced a series of mechanical models of the ether which are essentially conserving spatial discretizations of the continuum Maxwell equations. In these models a series of wheels are connected by elastic bands [21]. The rotational motion of the wheels is equivalent to the magnetic field while the extension, $\mathbf{E}$ of the elastic bands is equivalent to the electric field. If we perform Monte-Carlo on FitzGerald's wheels we again recover our local algorithm for Monte-Carlo simulation.

\section{References}

[1] T. Schlick, R. D. Skeel, A. T. Brunger, L. V. Kalé, J. A. Board, Jr., J. Hermans, K. Schulten, Algorithmic challenges in computational molecular biophysics, J. Comp. Phys. 151 (1999) 9-48.

[2] U. Essmann, L. Perera, M. L. Berkowitz, T. Darden, H. Lee, L. G. Pedersen, A smooth particle mesh ewald method, J. Chem. Phys. 103 (19) (1995) 8577-8593.

[3] J. Villasenor, O. Buneman, Rigorous charge conservation for local electro-magnetic field solver, Comp. Phys. Comm. 69 (2-3) (1992) 306-316.

[4] C. Birdsall, A. Langdon, Plasma Physics Via Computer Simulation, Institute of Physics Publishing, 1991.

[5] E. Hairer, C. Lubich, G. Wanner, Geometric Numerical Integration: Structure Preserving Algorithms for Ordinary Differential Equations, Springer-Verlag, 2002.

[6] C.-D. Munz, P. Omnes, R. Schneider, E. Sonnendrücker, U. Voss, Divergence correction techniques for maxwell solvers based on a hyperbolic model., J. Comput. Phys. 161 (2) (2000) 484-511.

[7] C.-D. Munz, R. Schneider, E. Sonnendrücker, U. Voss, Maxwell's equations when the charge conservation is not satisfied, C. R. Acad. Sci. Paris Sér. I Math. 328 (5) (1999) 431-436.

[8] J. Rottler, A. C. Maggs, A continuum, o(n) montecarlo algorithm for charged particles, J. Chem. Phys. 120 (7) (2004) 3119-3129.
[9] C. Sagui, T. Darden, Multigrid methods for classical molecular dynamics simulations of biomolecules, J. Chem. Phys. 114 (15) (2001) 6578-6591.

[10] M. Deserno, C. Holm, How to mesh up ewald sums. i. a theoretical and numerical comparison of various particle mesh routines, J. Chem. Phys. 109 (18) (1998) 7678-7693.

[11] J. Rottler, A. C. Maggs, Local molecular dynamics with coulombic interaction, cond-mat/0312438 .

[12] R. Car, M. Parrinello, Unified approach for molecular dynamics and density-functional theory, Phys. Rev. Lett. 55 (22) (1985) 2471-2474.

[13] S. J. Plimpton, R. Pollock, M. Stevens, Particlemesh ewald and rrespa for parallel molecular dynamics simulations, Proc of the Eighth SIAM Conference on Parallel Processing for Scientific Computing .

[14] I. Pasichnyk, B. Duenweg, Coulomb interactions via local dynamics: A molecular-dynamics algorithm, cond-mat/0406223 .

[15] J. S. Schwinger, L. L. Deraad, K. A. Milton, W. yang Tsai, Classical Electrodynamics, Perseus Books, 1998.

[16] A. C. Maggs, V. Rossetto, Local simulation algorithms for coulomb interactions, Phys. Rev. Lett. 88 (19) (2002) 196402.

[17] A. C. Maggs, Dynamics of a local algorithm for simulating coulomb interactions, J. Chem. Phys. 117 (5) (2002) 1975-1981.

[18] A. C. Maggs, Auxiliary field monte-carlo for charged particles, J. Chem. Phys. 120 (7) (2004) 3108-3118.

[19] F. Alet, E. S. Sorensen, Cluster monte-carlo algorithm for the quantum rotor model, Phys. Rev. E 67 (1) (2003) 015701.

[20] L. Levrel, F. Alet, J. Rottler, A. C. Maggs, Local simulation algorithms for coulombic interactions, Proceedings Statphys 22 (2004) .

URL http:/hogarth.pct.espci.fr/ ${ }^{\sim}\{\}$ tony/elec/sp7.pdf

[21] O. Darrigol, Electrodynamics from Ampère to Einstein, Oxford University Press, 2000. 